\title{
Genome analysis of Getah virus (GETV) isolated from a live swine vaccine: The commercial live vaccines might be a potential route for GETV transmission
}

\author{
Feng Zhou ${ }^{1}$, Aojie Wang ${ }^{1}$, Lu Chen ${ }^{1}$, Xingang Wang ${ }^{1}$, Dandan Cui ${ }^{1}$, Hongtao Chang ${ }^{1}$, and \\ chuan-qing WANG ${ }^{2}$ \\ ${ }^{1}$ Henan Agricultural University \\ ${ }^{2}$ Henan Aricultural University
}

May 18, 2020

\begin{abstract}
We reported a GETV strain, named GETV-V1, was isolated from a commercial modified live vaccine (MLV) against porcine reproductive and respiratory syndrome virus (PRRSV), which is widely used on pigs in China. Further results showed that nine batches of MLV vaccine (three batches per year) from the same manufacturer between 2015 and 2017 were all positive for GETV. The complete genomes of the isolate were sequenced and it was found that the genomes of GETV-V1 had the highest similarities with a strain 16-I-674, which was isolated from the sick horses in Japan. This is the first report of GETV contaminant in live swine vaccine in China. Our finding demonstrates that vaccination of the commercial live vaccines might be contaminated and became a potential new route for GETV transmission in swine, pointing to the need for more extensive monitoring of the commercial live vaccines in China.
\end{abstract}

\begin{abstract}
We reported a GETV strain, named GETV-V1, was isolated from a commercial modified live vaccine (MLV) against porcine reproductive and respiratory syndrome virus (PRRSV), which is widely used on pigs in China. Further results showed that nine batches of MLV vaccine (three batches per year) from the same manufacturer between 2015 and 2017 were all positive for GETV. The complete genomes of the isolate were sequenced and it was found that the genomes of GETV-V1 had the highest similarities with a strain 16-I674 , which was isolated from the sick horses in Japan. This is the first report of GETV contaminant in live swine vaccine in China. Our finding demonstrates that vaccination of the commercial live vaccines might be contaminated and became a potential new route for GETV transmission in swine, pointing to the need for more extensive monitoring of the commercial live vaccines in China.
\end{abstract}

KEY WORDS: Porcine reproductive and respiratory syndrome (PRRS) vaccine; Getah virus (GETV) contamination; Isolation;; Public health security

Getah virus (GETV) belongs to the genus Alphavirus within the family Togaviridae and is a mosquitotransmitted arbovirus (Berge et al., 1975) that can infect many vertebrates and arthropods (Doherty et al., 1966). The first GETV strain was isolated from Culex in Malaysia in 1955(Go et al., 2014). The prototype virus strain was MM2021 (Elisberg et al., 1963). Serological survey of GETV showed that GETV antibodies have been identified in humans, pigs, cattle, horses, goats, rabbits, kangaroos, chickens, foxes and some wild birds in many countries within Europe, Asia and Oceania. GETV can cause reproduction disorders and fetal death in pigs, as well as body rash, leg edema and fever in horses. The potential risk to animal health posed 
by GETV must not be overlooked (Li et al., 1992; Kurogi et al., 1975; Doherty et al., 1972; Tajima et al., 2014; Yago et al., 1987; Fukunaga et al., 2000)

The first GETV strain in China was isolated from mosquitoes in Hainan province in 1964. Since then, GETVs have been isolated from mosquitoes in more than 10 provinces in China, including Hainan, Shanghai, Sichuan, Yunnan, Hubei and Gansu (Yang et al., 1984; Li et al., 2017; Li et al., 2017; Li et al., 1992).

Porcine reproductive and respiratory syndrome virus (PRRSV) and GETV are different but common viruses associated with reproduction disorders in sows, and both are capable of growing in Marc-145 cells. PRRSV modified live vaccine (MLV) is widely used in China. In October 2017, in titer test of the PRRSV MLV vaccine used by a pig farm where abortion in a pregnant sow occurred, we found that the caused cytopathic effect (CPE) by the supernatant of vaccine on Marc-145 cells was significantly different from that of the previously vaccinated PRRSV strain. Similarly, CPE was also observed in other cell lines, including Vero, PK-15, BHK21 and human hepatocellular carcinoma HepG-2 cells. Therefore, exogenous virus was detected by reverse transcription-polymerase chain reaction (RT-PCR) using specific primers. The results demonstrated that GETV were positive. Because the pig farm had also been vaccinated with classical swine fever virus, Japanese encephalitis virus, porcine parvovirus and PRRSV. We suspected that GETV might be a contaminant in these commercial vaccines. As expected, a GETV strain was isolated from the commercial PRRS MLV vaccine following isolation, passage, purification by plaque assay and observation by transmission electron microscopy. This virus was designated as GETV-V1. In this study, we characterized the isolated GETV strain and the genome data of this newly identified GETV strain.

Further RT-PCR results showed that the commercial PRRS MLV vaccine was positive for GETV, including nine batches of vaccine (three batches per year) from the same manufacturer between 2015 and 2017. Immunofluorescence assay (Figure 1) showed that Marc-145 cells infected with the GETV isolate reacted specifically with GETV-specific monoclonal antibody (produced by our laboratory). The fairly uniform morphological appearance of GETV indicated that the diameter of the virus was 60-80 nm and close to spherical in shape. The fiber protein was clearly visible on the membrane (Figure 2). These data demonstrated that GETV-V1 was isolated from a contaminated live swine vaccine.

Excluding the poly(A) tail, the complete genome sequence of GETV-V1 was 11,689 nucleotides (nt) in length with a 5'-UTR of $78 \mathrm{nt}$ and 3'-UTR of $402 \mathrm{nt}$ (Table 1). The genome was similar to that of previously reported GETV strains. Sequence alignment of GETV-V1 with all GETV sequences available in GenBank revealed that GETV-V1 shared the highest identity (98.9\%) with 16-I-674 (isolated from a horse in Japan in 2016), 98.1\% identity with Chinese strains JL1707, JL1708, 12IH26 and Japanese strain 14-I-605-C2. Phylogenetic analysis showed that GETV-V1, together with HB0234, JL1707, JL1708, HNJZ-S1 and HNJZ-S2 strains, was clustered into the Japanese GETV branch (Fig.3). Interestingly, although the Chinese reference strains were close to GETV-V1 geographically and temporally, they were phylogenetically much closer to each other, compared with GETV-V1. These results revealed that the GETV strains circulating in China are genetically diverse, thus providing a potential platform for evolution.

Analysis using MegAlign software showed that GETV-V1 had 36 unique nucleotide mutations in its genome, including 35 nucleotide substitutions and 1 nucleotide insertion (Table 2). Interestingly, only 15 nucleotide mutations resulted in amino acid substitutions. These mutations with the nucleotide insertion in the 3' UTR of the genome could be a unique hallmark of GETV-V1.

In summary, we isolated and characterized one GETV strain from a commercial live pig vaccine produced in China. Complete genome analysis showed that GETV-V1 share high identity with GETV isolates HNJZ-S1, 14-I-605-C1 and 16-I-599, and these strains are pathogenic to pigs or horses (Nemoto et al., 2016). This study firstly reported for the contamination with GETV in live attenuated PRRS vaccines in China, implying that monitoring of exogenous virus in live vaccines for pigs needs to be improved. Furthermore, we should also pay more attention to the potential public health effect of vaccines contaminated with this virus.

\section{Acknowledgements}


The study was supported by the National Key Research and Development Program of China (NO 2016YFD0501100) and the Program for Science \& Technology Innovation Research Team in the Universities of Henan Province (NO 14IRTSTHN015)

\section{Conflict of interest statement}

The authors declare no conflict of interest.

\section{Ethics Statements}

All the sample collections were performed after obtaining verbal approval of farm owner. This study was approved by the Ethics Review Committee of the National Institute for Viral Disease Control and Prevention, Chinese Center for Disease Control and Prevention. All the animal studies were conducted in accordance with the principles of Henan agricultural university Animal Care and Use Committee (IACUC).

\section{Data Availability Statement}

All data generated or analyzed during this study are included in the article.

\section{References}

Berge, T. O. (1975). International catalogue of arboviruses, including certain other viruses of vertebrates.

Go, Y. Y., Balasuriya, U. B., \& Lee, C. K. (2014). Zoonotic encephalitides caused by arboviruses: transmission and epidemiology of alphaviruses and flaviviruses. Clinical and experimental vaccine research, 3(1), $58-77$.

Elisberg, B. L., \& Buescher, E. L. (1963). Arbovirus catalogue card No. 47. Arbovirus Information Exchange.

Doherty, R. L., Gorman, B. M., Whitehead, R. H., \& Carley, J. G. (1966). Studies of Arthropod-borne Virus Infections in Queensland: V. Survey of Antibodies to Group A Arboviruses in Man and other Animals. Australian Journal of Experimental Biology and Medical Science, 44(4), 365-378.

Li, X. D., Qiu, F. X., Yang, H., Rao, Y. N., \& Calisher, C. H. (1992). Isolation of Getah virus from mosquitos collected on Hainan Island, China, and results of a serosurvey. The Southeast Asian journal of tropical medicine and public health, 23(4), 730-734.

Kurogi, H., Inaba, Y., Goto, Y., Miura, Y., Takahashi, H., Sato, K., .. \& \& Matumoto, M. (1975). Serologic evidence for etiologic role of Akabane virus in epizootic abortion-arthrogryposis-hydranencephaly in cattle in Japan, 1972-1974. Archives of virology, 47(1), 71-83.

Doherty, R. L. (1972). Arboviruses of Australia. Australian veterinary journal, 48(4), 172-180.

Tajima, S., Kotaki, A., Yagasaki, K., Taniwaki, T., Moi, M. L., Nakayama, E., .. \& Takasaki, T. (2014). Identification and amplification of Japanese encephalitis virus and Getah virus propagated from a single porcine serum sample: a case of coinfection. Archives of virology, 159(11), 2969-2975.

Yago, K., Hagiwara, S., Kawamura, H., Narita, M.(1987). A fatal case in newborn piglets with Getah virus infection: isolation of the virus. Nihon Juigaku Zasshi, 49:989-94.

Fukunaga, Y., Kumanomido, T., \& Kamada, M. (2000). Getah virus as an equine pathogen. Veterinary Clinics of North America: Equine Practice , 16 (3), 605-617.

Yang, H., Rao, YN., Chen, RG., Hu.(1984). A strain of alphavirus togavviridae isolated in Hainan islandisolation, identification and aero-epidemic investigation. Chinese Journal of Microbiology and Immunology ,4(2):107.

Li, Y. Y., Fu, S. H., Guo, X. F., Lei, W. W., Li, X. L., Song, J. D., . . \& Wang, H. Y. (2017). Identification of a newly isolated Getah Virus in the China-Laos border, China. Biomedical and Environmental Sciences, 30(3), 210-214. 
Li, L., Guo, X., Zhao, Q., Tong, Y., Fan, H., Sun, Q., .. \& \& Zhang, J. (2017). Investigation on mosquitoborne viruses at Lancang river and Nu river watersheds in Southwestern China. Vector-Borne and Zoonotic Diseases, 17(12), 804-812.

Li, X. D., Qiu, F. X., Yang, H., Rao, Y. N., \& Calisher, C. H. (1992). Isolation of Getah virus from mosquitos collected on Hainan Island, China, and results of a serosurvey. The Southeast Asian journal of tropical medicine and public health, 23(4), 730-734.

Nemoto, M., Bannai, H., Tsujimura, K., Yamanaka, T., \& Kondo, T. (2016). Genomic, pathogenic, and antigenic comparisons of Getah virus strains isolated in 1978 and 2014 in Japan. Archives of virology, 161(6), 1691-1695.
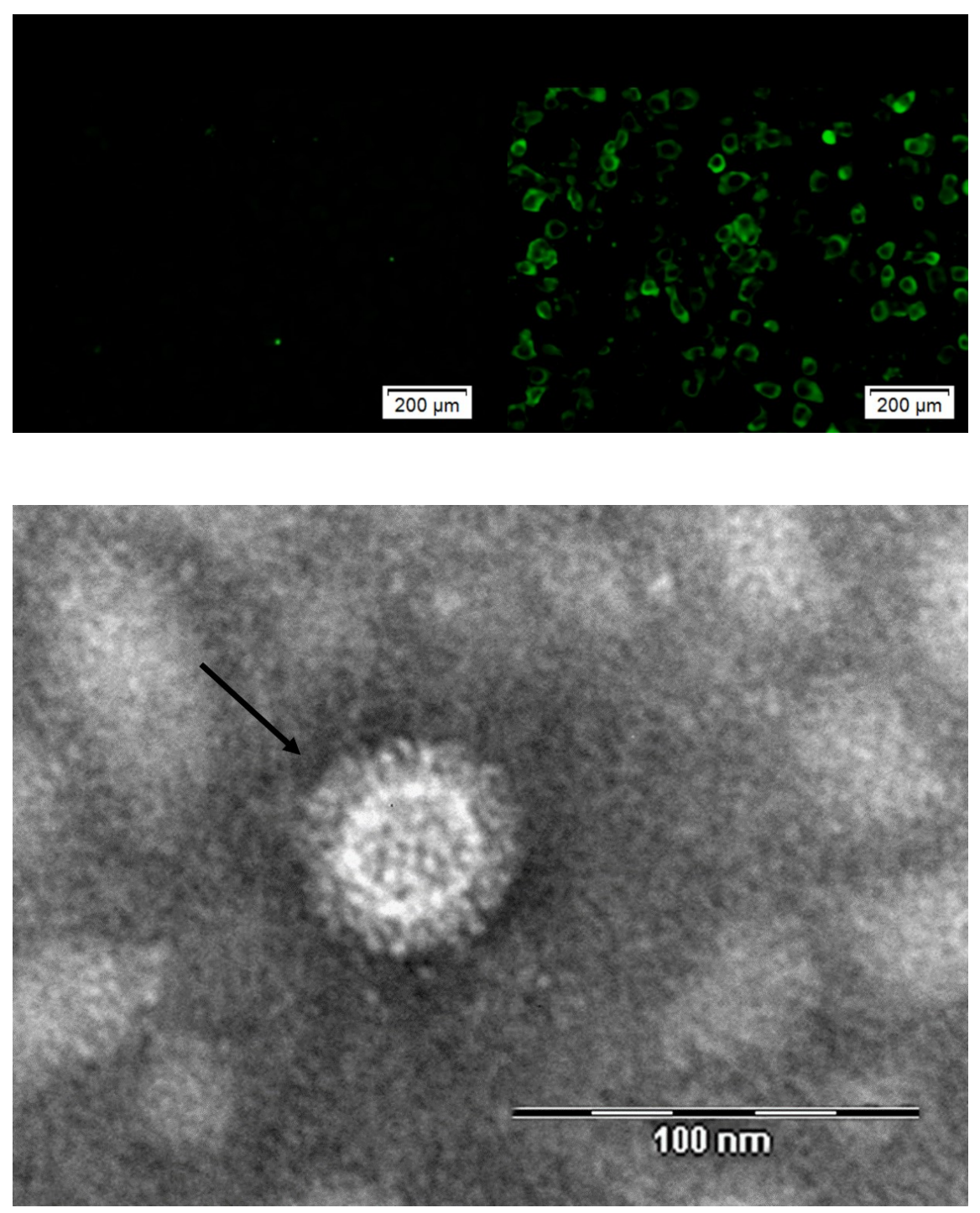


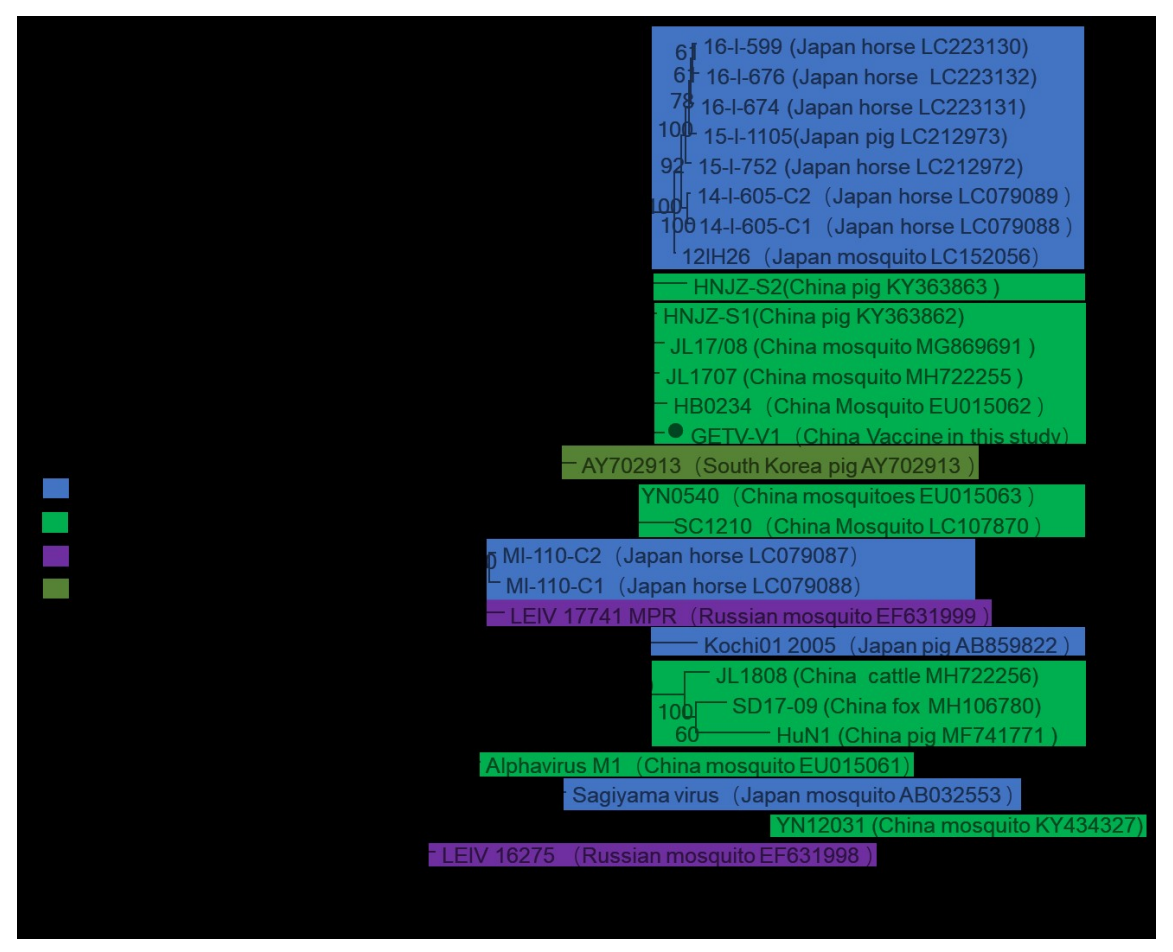

